\title{
Impact of timing of preoperative gabapentin administration on postoperative somnolence
}

https://doi.org/10.1515/jom-2021-0256

Received October 25, 2021; accepted January 25, 2022;

published online February 22, 2022

\begin{abstract}
Context: Enhanced Recovery After Surgery (ERAS) is a multimodal protocol aimed to improve quality of postoperative recovery, minimize complications, and optimize overall self-regulation. Preoperative gabapentin decreases postoperative pain but can be associated with prolonged postoperative somnolence and respiratory depression risk. Although it is known that gabapentin affects the postoperative course, it is unclear if the timing of preoperative administration affects this finding.

Objectives: This study aims to assess the optimal preoperative timing for gabapentin administration in patients undergoing gynecologic surgery to minimize postoperative somnolence risk.
\end{abstract}

Methods: A retrospective cohort study evaluated patients who underwent major gynecologic surgery and received preoperative gabapentin. Patients were grouped based on timing from gabapentin administration to surgical incision ( $<4 \mathrm{~h}$ group vs. $\geq 4 \mathrm{~h}$ group). Preoperative, intraoperative, and postoperative data were abstracted and compared. Univariate associations between the timing of gabapentin administration and the patient and surgical characteristics and outcomes were tested utilizing two-sample equalvariance t-tests, linear model ANOVA, or Fisher's exact tests. Associations between the timing of gabapentin administration and the time until the Richmond Agitation Sedation Scale (RASS) score of 0 were modeled utilizing linear regression, adjusted for age, initial postoperative

*Corresponding author: Summer Ghaith, BS, Mayo Clinic Alix School of Medicine, 6800 E. Mayo Blvd., Apt. 2412, Phoenix, AZ, USA,

E-mail: ghaith.summer@mayo.edu

Jaxon K. Quillen, MS, Carolyn Mead-Harvey, MS and Matthew R.

Buras, MS, Department of Quantitative Health Sciences, Mayo Clinic, Phoenix, AZ, USA

Megan N. Wasson, DO, Department of Medical and Surgical Gynecology, Mayo Clinic, Phoenix, AZ, USA anesthesia care unit (PACU), RASS score, and postoperative narcotics.

Results: Each group contained 127 patients. Demographics were similar except for age ( $<4 \mathrm{~h}$ group mean $=44.2$ years; $\geq 4 \mathrm{~h}$ group mean $=40.5$ years; $\mathrm{p}=0.021)$, chronic pain $(<4 \mathrm{~h}$ group=17.6\%; $\geq 4 \mathrm{~h}$ group=43.3\%; $<<0.001)$, and surgical indication $(<4 \mathrm{~h}$ group=pelvic pain $[29.1 \%] ; \geq 4 \mathrm{~h}$ group=pelvic pain [51.2\%]; $\mathrm{p}=0.007)$. The $<4 \mathrm{~h}$ group had a similar postoperative narcotic administration ( $<4 \mathrm{~h}$ group mean morphine milligram equivalents $[\mathrm{MME}]=3.667 ; \geq 4 \mathrm{~h}$ group mean $\mathrm{MME}=4.833 ; \mathrm{p}=0.185)$. The minutes from surgical closure until the patient received a RASS score of 0 and initial PACU pain score (Visual Analogue Scale [VAS]) were similar. The initial PACU oxygen administration volume, hours from surgical closure until the patient transitioned to room air, and initial PACU respiratory rate were similar. The PACU duration, admission secondary to somnolence, and initial PACU Glasgow Coma Scale (GCS) score showed no difference. Postoperative nausea/vomiting was decreased in the $\geq 4 \mathrm{~h}$ group ( $<4 \mathrm{~h}$ group $=24.4 \%$; $\geq 4 \mathrm{~h}$ group=13.4\%; $\mathrm{p}$-value $=0.036)$, and urinary retention $(<4 \mathrm{~h}$ group $=14.2 \%$; $\geq 4 \mathrm{~h}$ group $=5.5 \%$; $\mathrm{p}$-value $=0.033$ ) was decreased in the $\geq 4 \mathrm{~h}$ group.

Conclusions: The timing of gabapentin administration less than or more than $4 \mathrm{~h}$ preoperatively in patients $\geq 18$ years does not significantly affect postoperative somnolence or respiratory depression. Further, it does not have a significant effect on GCS scores or VAS scores.

Keywords: ERAS; gabapentin; hysterectomy; respiratory depression; somnolence.

Enhanced Recovery After Surgery (ERAS) is a multimodal protocol aimed to improve the quality of postoperative recovery and minimize complications [1]. The effectiveness of ERAS protocols have been assessed through analyzing patients' average length of stay and complications postoperatively [1]. ERAS supports the tenets of osteopathic medicine, including the promotion of self-regulation and self-healing, through preemptive management and optimization of anticipated intraoperative and postoperative physiologic changes [2]. The treatment employed in ERAS 
supports the interrelationship between all aspects of the body unit. Elements of ERAS protocols include, but are not limited to, patient education, patient optimization prior to admission, timing of oral intake of fluids and solids, nausea/vomiting prophylaxis, multimodal analgesia, and day of surgery ambulation [3-5].

ERAS protocols have narcotic-sparing effects by implementing multimodal analgesia with the incorporation of preemptive analgesia, systemic medications, and regional and neuraxial analgesia [4]. Acetaminophen, gabapentinoids, and nonsteroidal anti-inflammatory drugs are examples of non-narcotic drugs administered preoperatively to decrease narcotic consumption [3]. Specifically, preoperative gabapentin has shown to have narcotic-sparing effects and decreases postoperative pain in patients undergoing major gynecologic surgery [6-11]. Furthermore, patients who underwent minimally invasive gynecologic surgery who were administered gabapentin were found to have earlier ambulation, higher patient satisfaction, and earlier discharge from the hospital [11-13].

Gabapentin is an anticonvulsant that binds calciumdependent ion channels with antinociceptive and antihyperalgesic properties [9]. Specifically, gabapentin is utilized for chronic pain syndromes, such as neuropathic pain, and for seizure control $[9,10,14]$. Side effects include dizziness, diplopia, and ataxia [4]. When utilized perioperatively, it has also been associated with increased postoperative somnolence $[9,15]$ and respiratory depression [16]. Specifically, some ERAS protocols have discontinued the use of gabapentin in their protocols due to the Food and Drug Administration warning of respiratory depression from gabapentinoids, specifically when taken with central nervous system depressants and for geriatric patients or patients with renal impairments [17]. Gabapentin alone does not cause respiratory depression; however, in combination with opioids and specific patient characteristics, there is some evidence of respiratory depression [16]. Because of this potential interaction leading to adverse outcomes, some ERAS protocols have suspended the use of gabapentin.

Although it is known that gabapentin affects the postoperative course, it is unclear if the timing of preoperative administration affects this finding. The peak plasma concentration of gabapentin is known to occur approximately $2-3 \mathrm{~h}$ after administration [18]. This study aims to assess the optimal preoperative timing for gabapentin administration in patients undergoing gynecologic surgery to minimize the risk of prolonged postoperative somnolence. The primary outcome was measured by the time from surgical closure to a Richmond Agitation Sedation Scale (RASS) score of 0 . Secondary outcomes included the postoperative anesthesia care unit (PACU) stay duration, unplanned admission, and endpoints related to sedation, respiration, and pain.

\section{Methods}

After obtaining approval from the Mayo Clinic Institutional Review Board (IRB number: 20-003782), a retrospective cohort chart review was completed. The electronic health records (EHRs) of patients $\geq 18$ years old who received preoperative gabapentin and underwent major gynecologic surgery (benign, oncologic, and urogynecologic procedures) between March 2018 and November 2020 were identified. Major gynecologic surgery was defined as a surgical procedure that required entrance into the peritoneal cavity (laparotomy, laparoscopy, and robotic and vaginal surgeries). Patients were grouped based on the timing from gabapentin administration to surgical incision (less than $4 \mathrm{~h}$ vs. four or more hours). Medical, surgical, and anesthesia records were reviewed. Preoperative, intraoperative, and postoperative data were abstracted and compared.

The demographic data abstracted included age, weight, height, menopause status, race/ethnicity, chronic narcotic use, chronic gabapentin use, and chronic pain status. Race/ethnicity was selfdesignated by patients utilizing the hospital-predetermined classifications of: American Indian or Alaska Native; Asian; Black or African American; Hispanic or Latino; Native Hawaiian or Other Pacific Islander; White; or Other. Race/ethnicity were assessed solely to describe the patient demographics in this study. The surgical data abstracted included surgical indication, surgical approach, procedure performed, time of first incision, time of closure, surgical duration, and estimated blood loss. The time of the gabapentin administration was recorded. Per institutional ERAS protocol, patients aged 18-59 were given $600 \mathrm{mg}$ of gabapentin, and patients aged 60 years and older received $300 \mathrm{mg}$ of gabapentin. Preoperative, intraoperative, and postoperative narcotic consumption were abstracted. Narcotic administration was converted to IV morphine milligram equivalents (MME) [19]. The PACU data abstracted included variables that were utilized collectively as surrogate markers of somnolence. These included vital signs, RASS scores, oxygen administration, Glasgow Coma Scale (GCS) scores, unplanned hospital admission, dizziness, nausea/vomiting, urinary retention, and duration of PACU stay. Postoperative nausea and vomiting were recorded if they were subjectively reported in the patient's medical record.

A sample size of 254 patients with 127 patients in each group was estimated to provide power of $80 \%$ or greater, assuming a mean somnolence recovery time of $90 \mathrm{~min}$ in the more than or equal to $4 \mathrm{~h}$ gabapentin group, $150 \mathrm{~min}$ in the less than $4 \mathrm{~h}$ gabapentin group, a standard deviation of $150 \mathrm{~min}$, and a significance level of 5\%. This sample size allowed for a missing data rate of $10 \%$. Patients were identified starting in March 2018 and continued until 127 patients were reached in each group. Patients were excluded if they did not have both a gynecologic surgery and preoperative gabapentin administration.

The data were analyzed to determine if there was a difference in patient-oriented outcomes among individuals taking gabapentin within $4 \mathrm{~h}$ of surgery or more than $4 \mathrm{~h}$ of surgery. This cutoff was based on the typical time intervals with patients self-administering gabapentin at home vs. receiving gabapentin in the preoperative unit. Associations were tested utilizing two-sample equal-variance t-tests or 
linear model ANOVA for continuous variables, or Fisher's exact tests for categorical variables. Associations between the time from taking gabapentin to the time of first incision and the time until RASS score of 0 were modeled utilizing linear regression, adjusting for age, initial PACU RASS score, and postoperative narcotics (MME). Statistical analysis was performed utilizing $R$ version 4.0.3.

\section{Results}

A total of 254 patients were included. Gabapentin was administered less than $4 \mathrm{~h}$ prior to skin incision for 127 patients (50.0\%) ( $<4 \mathrm{~h}$ group) and four or more hours in 127 patients (50.0\%) ( $\geq 4 \mathrm{~h}$ group). Demographics were similar between the groups with the exception of age $(<4 \mathrm{~h}$ group mean $=44.2 ; \geq 4 \mathrm{~h}$ group=mean $40.5 ; \mathrm{p}=0.021$ ), chronic pain ( $<4 \mathrm{~h}$ group $=17.6 \%, \mathrm{n}=22 ; \geq 4 \mathrm{~h}$ group $=43.3 \%, \mathrm{n}=55$; $\mathrm{p}<0.001)$, and surgical indication $(<4 \mathrm{~h}$ group $=$ pelvic pain [29.1\%, $\mathrm{n}=37$ ]; $\geq 4 \mathrm{~h}$ group=pelvic pain $(51.2 \%, \mathrm{n}=65)$; $\mathrm{p}=0.007)$. Surgical approach was minimally invasive in $78.0 \%(n=99)$ of patients in $<4$ h group and $88.2 \%(n=112)$ of patients in $\geq 4 \mathrm{~h}$ group. Hysterectomy was the most frequent procedure performed ( $<4 \mathrm{~h}$ group $=50.4 \%, \mathrm{n}=64 ; \geq 4 \mathrm{~h}$ group $=49.6 \%, n=63)$, followed by excision of endometriosis ( $<4 \mathrm{~h}$ group $=27.6 \%, \mathrm{n}=35$; $\geq 4 \mathrm{~h}$ group $=55.1 \%, \mathrm{n}=70$ ). Details of demographics and surgical characteristics are summarized in Table 1.

Narcotic administration preoperatively $(\mathrm{p}=0.111)$ and intraoperatively $(p=0.237)$, and postoperatively $(p=0.185)$ were similar between the two groups. Oxycodone and hydromorphone were administered less frequently in the $<4 \mathrm{~h}$ group (31.5\%, $\mathrm{n}=40$ and $29.9 \%, \mathrm{n}=38$, respectively) than in the $\geq 4$ h group $(48.8 \%, n=62$ and $44.9 \%, n=57)(p=0.007$ and 0.019 respectively). Preoperative, intraoperative, and postoperative medications are outlined in Table 2.

The minutes from surgical closure until the time the patient received a RASS score of $0(<4 \mathrm{~h}$ group mean [SD] $=163.5[117.9] \mathrm{min}, \geq 4 \mathrm{~h}$ group mean $[\mathrm{SD}]=143.9$ [100.4] $\mathrm{min}$, $\mathrm{p}=0.168$ ) and initial PACU pain score (VAS) were similar for both groups $(\mathrm{p}=0.245)$. The initial PACU oxygen administration volume $(\mathrm{p}=0.678)$, hours from surgical closure until the patient transitioned to room air $(\mathrm{p}=0.679)$, and initial PACU respiratory rate $(\mathrm{p}=0.913)$ were similar between the two groups. The mean PACU stay duration did not show a statistical difference with the less than $4 \mathrm{~h}$ group duration of stay of $250.1 \mathrm{~min}$ compared to $240.1 \mathrm{~min}$ in the more than $4 \mathrm{~h}$ group $(\mathrm{p}=0.449)$. Unplanned admission secondary to excessive somnolence was uncommon in both groups, with one instance $(0.8 \%)$ in the less than $4 \mathrm{~h}$ group and two instances $(1.6 \%)$ in the more than $4 \mathrm{~h}$ group $(\mathrm{p}=1.00)$. The initial PACU GCS score $(p=0.473)$ showed no statistically significant difference between the two groups. Postoperative dizziness had no significant difference between the two groups; however, postoperative nausea/vomiting ( $<4 \mathrm{~h}$ group $=24.4 \%, \mathrm{n}=31 ; \geq 4 \mathrm{~h}$ group $=13.4 \%, \mathrm{n}=17$; $\mathrm{p}=0.036)$ and urinary retention $(<4 \mathrm{~h}$ group $=14.2 \%$, $\mathrm{n}=18$; $\geq 4 \mathrm{~h}$ group $=5.5 \%, \mathrm{n}=7 ; \mathrm{p}=0.033$ ) were both decreased in the $4 \mathrm{~h}$ or more group. Table 3 compares the postoperative clinical outcomes by time of taking gabapentin to time of first incision.

After controlling for age, initial PACU RASS score, and postoperative narcotics use, patients took on average $0.105 \mathrm{~min}$ less (95\% CI: $0.232 \mathrm{~min}$ less to $0.021 \mathrm{~min}$ more) to achieve a RASS score of 0 for every increase of $1 \mathrm{~min}$ between taking gabapentin to the time of first incision $(p=0.10)$. However, this finding was not statistically significant. Controlling for time from taking gabapentin to time of first incision, initial PACU RASS score, and postoperative narcotics use, patients took on average $2.983 \mathrm{~min}$ more (95\% CI: $2.675 \mathrm{~min}$ less to $8.640 \mathrm{~min}$ more) to achieve a RASS score of 0 for every five-year increase in age, although this difference was not statistically significant $(\mathrm{p}=0.30)$. Results for the linear regression for the outcome of time until RASS score of 0 are outlined in Table 4.

Minutes from closure until time achieved a RASS score of 0 stratified by indication of surgery showed the indication of abnormal bleeding took the greatest amount of time (median, 155). This was followed by pelvic pain (median, 134), cancer (median, 129), other (median, 128), and finally, prolapse (median, 95.50) ( $\mathrm{p}=0.85)$. Table 5 reports the summary of time until RASS score of 0 by surgical indication.

\section{Discussion}

This retrospective chart review demonstrated that the timing of preoperative gabapentin administration as part of the ERAS protocol prior to gynecologic surgeries does not have a significant effect on postoperative somnolence, GCS scores, or pain VAS scores. Further, this study found that gabapentin does not have a significant effect on respiratory depression when given as part of the ERAS protocol for gynecologic surgeries. This finding is important in light of a recent study stating that some ERAS protocols are discontinuing the use of gabapentin due to the risk of respiratory depression [17].

This study shows that the timing of gabapentin does not affect postoperative somnolence. Previous studies have found an increased risk of sedation with preoperative gabapentin $[9,15]$. It has been hypothesized that receiving gabapentin four or more hours prior to surgery would decrease the risk of postoperative sedation. However, this 
Table 1: Demographics and surgical characteristics by time from taking gabapentin to time of first incision.

\begin{tabular}{|c|c|c|c|}
\hline & $<4 h(n=127)$ & $\geq 4 h(n=127)$ & p-Value \\
\hline Age, years & & & 0.021 \\
\hline Mean, SD & $44.2(13.3)$ & $40.5(12.1)$ & \\
\hline Median (Q1, Q3) & $43.0(35.0,53.0)$ & $38.0(31.0,47.0)$ & \\
\hline Range & $20.0-83.5$ & $20.0-69.0$ & \\
\hline Weight, kg & & & 0.414 \\
\hline Mean, SD & $75.5(18.5)$ & $73.6(18.8)$ & \\
\hline Median (Q1, Q3) & $72.1(63.1,84.1)$ & $68.8(59.5,85.5)$ & \\
\hline Range & $43.8-182.0$ & $41.5-142.0$ & \\
\hline Height, $\mathrm{cm}$ & & & 0.050 \\
\hline Mean, SD & $165.2(6.3)$ & $166.7(6.4)$ & \\
\hline Median (Q1, Q3) & $165.0(161.0,170.2)$ & $166.4(162.7,169.9)$ & \\
\hline Range & $152.0-181.9$ & $149.7-184.2$ & \\
\hline Body mass index, $\mathrm{kg} / \mathrm{m}^{2}$ & & & 0.122 \\
\hline Mean, SD & $27.7(6.7)$ & $26.4(6.4)$ & \\
\hline Median (Q1, Q3) & $26.2(23.4,31.5)$ & $25.1(21.7,30.0)$ & \\
\hline Range & $16.8-66.8$ & $14.9-49.0$ & \\
\hline Premenopausal & $86(67.7 \%)$ & $96(75.6 \%)$ & 0.210 \\
\hline \multicolumn{4}{|l|}{ Race/ethnicity } \\
\hline American Indian or Alaska Native & $0(0.0 \%)$ & $0(0.0 \%)$ & \\
\hline Asian & $5(3.9 \%)$ & $7(5.5 \%)$ & \\
\hline Black or African American & $8(6.3 \%)$ & $4(3.1 \%)$ & \\
\hline Hispanic or Latino & $1(0.8 \%)$ & $11(8.7 \%)$ & \\
\hline Native Hawaiian or other Pacific Islander & $0(0.0 \%)$ & $0(0.0 \%)$ & \\
\hline White & $106(83.5 \%)$ & $101(79.5 \%)$ & \\
\hline Other & $7(5.5 \%)$ & $4(3.1 \%)$ & \\
\hline Chronic narcotic use & $3(2.4 \%)$ & $4(3.2 \%)$ & 0.721 \\
\hline Chronic pain & $22(17.6 \%)$ & $55(43.3 \%)$ & $<0.001$ \\
\hline Chronic gabapentin use & $4(3.1 \%)$ & $8(6.3 \%)$ & 0.254 \\
\hline Surgical indication & & & 0.007 \\
\hline Pelvic pain & $37(29.1 \%)$ & $65(51.2 \%)$ & \\
\hline Cancer & $18(14.2 \%)$ & $10(7.9 \%)$ & \\
\hline Prolapse & $15(11.8 \%)$ & $8(6.3 \%)$ & \\
\hline Abnormal bleeding & $23(18.1 \%)$ & $20(15.7 \%)$ & \\
\hline Other & $34(26.8 \%)$ & $24(18.9 \%)$ & \\
\hline Type of procedure & & & $<0.001$ \\
\hline Robotic & $59(46.5 \%)$ & $95(74.8 \%)$ & \\
\hline Laparoscopic & $40(31.5 \%)$ & $17(13.4 \%)$ & \\
\hline Vaginal & $22(17.3 \%)$ & $7(5.5 \%)$ & \\
\hline Laparotomy & $6(4.7 \%)$ & $8(6.3 \%)$ & \\
\hline \multicolumn{4}{|l|}{ Procedure performed } \\
\hline Hysterectomy & $64(50.4 \%)$ & $63(49.6 \%)$ & 1.000 \\
\hline Excision of endometriosis & $35(27.6 \%)$ & $70(55.1 \%)$ & $<0.001$ \\
\hline Cancer staging & $11(8.7 \%)$ & $2(1.6 \%)$ & 0.019 \\
\hline Ovarian cystectomy & $18(14.2 \%)$ & $13(10.2 \%)$ & 0.444 \\
\hline Salpingectomy & $59(46.5 \%)$ & $58(45.7 \%)$ & 1.000 \\
\hline Salpingo-oophorectomy & $30(23.6 \%)$ & $17(13.4 \%)$ & 0.052 \\
\hline Prolapse repair & $14(11.0 \%)$ & $8(6.3 \%)$ & 0.264 \\
\hline Other & $60(47.2 \%)$ & $81(63.8 \%)$ & 0.011 \\
\hline Duration of procedure, $h$ & & & 0.043 \\
\hline Mean, SD & $2.1(1.3)$ & $2.4(1.4)$ & \\
\hline Median (Q1, Q3) & $1.8(1.1,2.7)$ & $2.2(1.5,2.9)$ & \\
\hline Range & $0.5-8.4$ & $0.4-9.8$ & \\
\hline $\mathrm{EBL}, \mathrm{mL}$ & & & 0.434 \\
\hline Mean, SD & 93.9 (199.6) & $78.0(112.7)$ & \\
\hline Median (Q1, Q3) & $50.0(20.0,95.0)$ & $50.0(20.0,100.0)$ & \\
\hline Range & $0.0-1800.0$ & $5.0-750.0$ & \\
\hline
\end{tabular}

SD, standard deviation. 
Table 2: Preoperative, intraoperative, and postoperative medications by time from taking gabapentin to time of first incision.

\begin{tabular}{|c|c|c|c|}
\hline & $<4 h(n=127)$ & $\geq 4 h(n=127)$ & p-Value \\
\hline Preoperative narcotics, $\mathrm{MME}$ & & & 0.111 \\
\hline Mean, SD & $0.220(0.778)$ & $0.424(1.207)$ & \\
\hline Median (Q1, Q3) & $0.025(0.013,0.025)$ & $0.025(0.016,0.025)$ & \\
\hline Range & $0.000-4.025$ & $0.000-7.525$ & \\
\hline Narcotic meds: dilaudid & $0(0.0 \%)$ & $0(0.0 \%)$ & \\
\hline Narcotic meds: oxycodone & $0(0.0 \%)$ & $1(0.8 \%)$ & 1.000 \\
\hline Narcotic meds: hydrocodone & $0(0.0 \%)$ & $0(0.0 \%)$ & \\
\hline Narcotic meds: morphine & $1(0.8 \%)$ & $0(0.0 \%)$ & 1.000 \\
\hline Narcotic meds: fentanyl & $122(96.1 \%)$ & $122(96.1 \%)$ & 1.000 \\
\hline Narcotic meds: hydromorphone & $8(6.3 \%)$ & $15(11.8 \%)$ & 0.189 \\
\hline Narcotic meds: oxymorphone & $0(0.0 \%)$ & $0(0.0 \%)$ & \\
\hline Narcotic meds: tramadol & $0(0.0 \%)$ & $0(0.0 \%)$ & \\
\hline Intraoperative narcotics, MME & & & 0.237 \\
\hline Mean, SD & $0.695(1.393)$ & $0.901(1.376)$ & \\
\hline Median (Q1, Q3) & $0.013(0.000,0.025)$ & $0.013(0.000,2.000)$ & \\
\hline Range & $0.000-6.400$ & $0.000-4.025$ & \\
\hline Narcotic meds: dilaudid & $0(0.0 \%)$ & $0(0.0 \%)$ & \\
\hline Narcotic meds: oxycodone & $0(0.0 \%)$ & $0(0.0 \%)$ & \\
\hline Narcotic meds: hydrocodone & $0(0.0 \%)$ & $0(0.0 \%)$ & \\
\hline Narcotic meds: morphine & $0(0.0 \%)$ & $0(0.0 \%)$ & \\
\hline Narcotic meds: fentanyl & $68(53.5 \%)$ & $52(40.9 \%)$ & 0.059 \\
\hline Narcotic meds: hydromorphone & $31(24.4 \%)$ & $47(37.0 \%)$ & 0.041 \\
\hline Narcotic meds: oxymorphone & $0(0.0 \%)$ & $0(0.0 \%)$ & \\
\hline Narcotic meds: tramadol & $0(0.0 \%)$ & $0(0.0 \%)$ & \\
\hline Postoperative narcotics, MME & & & 0.185 \\
\hline Mean, SD & $3.667(8.054)$ & $4.833(5.726)$ & \\
\hline Median (Q1, Q3) & $0.019(0.006,7.506)$ & $4.006(0.006,8.306)$ & \\
\hline Range & $0.000-78.206$ & $0.000-46.606$ & \\
\hline Narcotic meds: dilaudid & $0(0.0 \%)$ & $0(0.0 \%)$ & \\
\hline Narcotic meds: oxycodone & $40(31.5 \%)$ & $62(48.8 \%)$ & 0.007 \\
\hline Narcotic meds: hydrocodone & $1(0.8 \%)$ & $0(0.0 \%)$ & 1.000 \\
\hline Narcotic meds: morphine & $2(1.6 \%)$ & $0(0.0 \%)$ & 0.498 \\
\hline Narcotic meds: fentanyl & 97 (76.4\%) & $96(75.6 \%)$ & 1.000 \\
\hline Narcotic meds: hydromorphone & $38(29.9 \%)$ & $57(44.9 \%)$ & 0.019 \\
\hline Narcotic meds: oxymorphone & $0(0.0 \%)$ & $0(0.0 \%)$ & \\
\hline Narcotic meds: tramadol & $1(0.8 \%)$ & $1(0.8 \%)$ & 1.000 \\
\hline
\end{tabular}

MME, morphine milligram equivalents; SD, standard deviation.

study shows no significant differences in RASS scores and duration of PACU stay between the two groups. Further, both groups achieved a RASS score of 0 within $2.4-2.7 \mathrm{~h}$, on average, after surgical closure. Both groups were discharged from the PACU within 4-4.17 h, on average, of surgical closure. The protocol at the study institution is to monitor patients following a hysterectomy for a minimum of $4 \mathrm{~h}$ in the PACU prior to discharge, indicating that, on average, patients were discharged as early as the protocol allowed. Between both groups, only three patients had an unplanned admission secondary to excessive somnolence. These findings suggest that the risk for postoperative somnolence may not be as high in patients receiving preoperative gabapentin in gynecologic surgeries as previous studies have shown $[9,15]$.

One study of 8,567 patients who underwent laparoscopic surgeries lasting 90 min or longer found that 15.3\% of the patients had episodes of respiratory depression when receiving gabapentin preoperatively [16]. In this study, respiratory depression was evaluated through respiratory rate, liters of oxygen needed, and hours from surgical closure until the patient was transitioned to room air. This study did not find evidence of complicated respiratory depression in either group, indicating that the timing of gabapentin does not cause differences in respiration postoperatively and further illustrates that the risk of 
Table 3: Main outcomes by time from taking gabapentin to time of first incision.

\begin{tabular}{|c|c|c|c|}
\hline & $<4 h(n=127)$ & $\geq 4 h(n=127)$ & p-Value \\
\hline Minutes from closure until RASS score of 0 & & & 0.168 \\
\hline Mean, SD & $163.5(117.9)$ & $143.9(100.4)$ & \\
\hline Median (Q1, Q3) & $142.5(77.8,205.8)$ & $128.0(67.5,193.5)$ & \\
\hline Range & $11.0-743.0$ & $8.0-708.0$ & \\
\hline Initial PACU RASS score & & & 0.675 \\
\hline Mean, SD & $-1.4(1.0)$ & $-1.4(1.1)$ & \\
\hline Median (Q1, Q3) & $-1.0(-1.0,-1.0)$ & $-1.0(-2.0,-1.0)$ & \\
\hline Range & $-5.0-1.0$ & $-5.0-3.0$ & \\
\hline $1 \mathrm{~h}$ postoperative RASS score & & & 0.006 \\
\hline Mean, SD & $-0.7(0.5)$ & $-0.6(0.6)$ & \\
\hline Median (Q1, Q3) & $-1.0(-1.0,0.0)$ & $-1.0(-1.0,0.0)$ & \\
\hline Range & $-2.0-0.0$ & $-2.0-1.0$ & \\
\hline Initial PACU pain score VAS & & & 0.245 \\
\hline Mean, SD & $2.6(3.3)$ & $3.1(3.3)$ & \\
\hline Median (Q1, Q3) & $0.0(0.0,5.0)$ & $2.0(0.0,6.0)$ & \\
\hline Range & $0.0-10.0$ & $0.0-10.0$ & \\
\hline $1 \mathrm{~h}$ postoperative pain score & & & 0.170 \\
\hline Mean, SD & $3.8(2.5)$ & $4.2(2.3)$ & \\
\hline Median (Q1, Q3) & $4.0(2.0,5.0)$ & $4.0(3.0,6.0)$ & \\
\hline Range & $0.0-9.0$ & $0.0-10.0$ & \\
\hline Initial PACU L of $\mathrm{O}_{2}$ & & & 0.678 \\
\hline Mean, SD & $7.3(2.1)$ & $7.2(1.8)$ & \\
\hline Median (Q1, Q3) & $8.0(6.0,8.0)$ & $8.0(6.0,8.0)$ & \\
\hline Range & $2.0-15.0$ & $2.0-10.0$ & \\
\hline \multicolumn{4}{|l|}{ Initial PACU route } \\
\hline Nasal cannula & $10(8.2 \%)$ & $6(4.8 \%)$ & \\
\hline Face mask & $111(91.0 \%)$ & $118(95.2 \%)$ & \\
\hline CPAP & $0(0.0 \%)$ & $0(0.0 \%)$ & \\
\hline ET tube & $1(0.8 \%)$ & $0(0.0 \%)$ & \\
\hline $1 \mathrm{~h}$ postop $\mathrm{L}$ of $\mathrm{O}_{2}$ & & & 0.586 \\
\hline Mean, SD & $2.6(1.6)$ & $2.5(1.2)$ & \\
\hline Median (Q1, Q3) & $2.0(2.0,3.0)$ & $2.0(2.0,3.0)$ & \\
\hline Range & $1.0-10.0$ & $1.0-10.0$ & \\
\hline \multicolumn{4}{|l|}{$1 \mathrm{~h}$ postoperative route } \\
\hline Nasal cannula & 44 (95.7\%) & $51(98.1 \%)$ & \\
\hline Face mask & $2(4.3 \%)$ & $1(1.9 \%)$ & \\
\hline CPAP & $0(0.0 \%)$ & $0(0.0 \%)$ & \\
\hline ET tube & $0(0.0 \%)$ & $0(0.0 \%)$ & \\
\hline Time from closure until patient on room air, $h$ & & & 0.679 \\
\hline Mean, SD & $2.4(2.7)$ & $2.3(2.8)$ & \\
\hline Median (Q1, Q3) & $1.2(0.7,3.4)$ & $1.4(0.7,2.7)$ & \\
\hline Range & $0.3-12.7$ & $0.1-17.7$ & \\
\hline Initial PACU respiratory rate & & & 0.913 \\
\hline Mean, SD & $15.5(4.5)$ & $15.5(4.0)$ & \\
\hline Median (Q1, Q3) & $15.0(12.5,18.0)$ & $15.5(13.0,17.0)$ & \\
\hline Range & $7.0-28.0$ & $5.0-28.0$ & \\
\hline $1 \mathrm{~h}$ postoperative respiratory rate & & & 0.176 \\
\hline Mean, SD & $15.0(4.0)$ & $15.7(3.4)$ & \\
\hline Median (Q1, Q3) & $15.0(12.0,17.0)$ & $15.0(13.0,18.0)$ & \\
\hline Range & $7.0-29.0$ & $7.0-26.0$ & \\
\hline PACU stay duration, min & & & 0.449 \\
\hline Mean, SD & $250.1(114.3)$ & $240.1(95.8)$ & \\
\hline Median (Q1, Q3) & $249.0(180.5,283.0)$ & $243.0(163.0,297.0)$ & \\
\hline Range & $70.0-713.0$ & $67.0-538.0$ & \\
\hline Unplanned admission secondary to excessive somnolence & & & 1.000 \\
\hline No & $124(99.2 \%)$ & $124(98.4 \%)$ & \\
\hline Yes & $1(0.8 \%)$ & $2(1.6 \%)$ & \\
\hline
\end{tabular}


Table 3: (continued)

\begin{tabular}{|c|c|c|c|}
\hline & $<4 h(n=127)$ & $\geq 4 h(n=127)$ & p-Value \\
\hline $\begin{array}{l}\text { Unplanned admission secondary to } \\
\text { reason unrelated to somnolence }\end{array}$ & & & 0.300 \\
\hline No & $109(87.2 \%)$ & $115(92.0 \%)$ & \\
\hline Yes & 16 (12.8\%) & 10 (8.0\%) & \\
\hline GCS (alertness) initial score & & & 0.473 \\
\hline Mean, SD & $13.9(1.8)$ & $13.6(2.1)$ & \\
\hline Median (Q1, Q3) & $14.0(14.0,15.0)$ & $14.0(14.0,15.0)$ & \\
\hline Range & $3.0-15.0$ & $6.0-15.0$ & \\
\hline GCS (alertness) $1 \mathrm{~h}$ postoperative & & & 0.463 \\
\hline Mean, SD & $14.8(0.4)$ & $14.7(1.0)$ & \\
\hline Median (Q1, Q3) & $15.0(15.0,15.0)$ & $15.0(15.0,15.0)$ & \\
\hline Range & $14.0-15.0$ & $11.0-15.0$ & \\
\hline Dizziness & $9(7.1 \%)$ & $7(5.5 \%)$ & 0.797 \\
\hline Nausea/vomiting & $31(24.4 \%)$ & $17(13.4 \%)$ & 0.036 \\
\hline Urinary retention & $18(14.2 \%)$ & $7(5.5 \%)$ & 0.033 \\
\hline
\end{tabular}

CPAP, continuous positive airway pressure; ET, endotracheal; GCS, glasgow coma scale; PACU, postoperative anesthesia care unit; RASS, richmond agitation sedation scale; SD, standard deviation; VAS, visual analog scale.

Table 4: Results from linear regression for the outcome of time until RASS score of 0 .

\begin{tabular}{lrrrr}
\hline & Estimate & $\begin{array}{r}\text { Cl lower } \\
\text { estimate }\end{array}$ & $\begin{array}{r}\text { Cl upper } \\
\text { estimate }\end{array}$ & p-Value \\
\hline Time from taking gaba- & -0.105 & -0.232 & 0.021 & 0.102 \\
pentin to time of first & & & & \\
$\begin{array}{l}\text { incision, min } \\
\text { Age, years }\end{array}$ & 2.983 & -2.675 & 8.640 & 0.300 \\
Initial PACU RASS score: & -12.379 & -26.169 & 1.411 & 0.078 \\
Postoperative & 1.474 & -0.507 & 3.455 & 0.144 \\
narcotics, MME & & & & \\
\hline
\end{tabular}

$\mathrm{Cl}$, confidence interval; MME, morphine milligram equivalents; PACU, postoperative anesthesia care unit; RASS, richmond agitation sedation scale.

postoperative respiratory depression may be smaller in the gynecologic surgery population than other studies have previously shown $[16,20]$.
This study found that the adverse outcomes of nausea and vomiting may be decreased when gabapentin is given more than $4 \mathrm{~h}$ prior to surgery. Other studies have found the incidence of postoperative nausea and vomiting to be decreased in patients receiving gabapentin [9, 21, 22]. A meta-analysis found a $40 \%$ relative risk reduction in postoperative nausea and vomiting in patients who received preoperative gabapentin [22]. This may be attributed to the innate anti-emetic effects [20] of gabapentin and the longer time for gabapentin to be distributed throughout the body. Gabapentin decreases nausea and vomiting symptoms by regulating efferent and afferent neural pathways involved in their production. It is increasingly being utilized in ERAS protocols and for chronic nausea [23]. Furthermore, this study found that incidents of urinary retention may be decreased when gabapentin is given more than $4 \mathrm{~h}$ preoperatively. A meta-analysis of 827 patients found that urinary retention was decreased in patients who received gabapentin

Table 5: Summary of time until RASS score of 0 by indication.

\begin{tabular}{|c|c|c|c|c|c|c|c|}
\hline & $\begin{array}{r}\text { Pelvic pain } \\
(n=102)\end{array}$ & $\begin{array}{l}\text { Cancer } \\
(n=28)\end{array}$ & $\begin{array}{r}\text { Prolapse } \\
(n=23)\end{array}$ & $\begin{array}{r}\text { Abnormal } \\
\text { bleeding }(n=44)\end{array}$ & $\begin{array}{r}\text { Other } \\
(n=58)\end{array}$ & $\begin{array}{r}\text { Total } \\
(n=255)\end{array}$ & p-Value \\
\hline $\begin{array}{l}\text { Minutes from } \\
\text { closure until } \\
\text { time achieved a } \\
\text { RASS score of } 0\end{array}$ & & & & & & & 0.845 \\
\hline Mean, SD & $159.88(124.53)$ & $138.40(78.59)$ & $144.95(116.93)$ & $161.80(92.74)$ & $146.18(104.30)$ & $153.47(109.48)$ & \\
\hline $\begin{array}{l}\text { Median (Q1, } \\
\text { Q3) }\end{array}$ & $\begin{array}{r}134.00 \\
(78.75,208.25)\end{array}$ & $\begin{array}{r}129.00 \\
(82.00,193.00)\end{array}$ & $\begin{array}{r}95.50 \\
(63.50,186.00)\end{array}$ & $\begin{array}{r}155.00 \\
(75.00,216.00)\end{array}$ & $\begin{array}{r}128.00 \\
(74.00,168.00)\end{array}$ & $\begin{array}{r}133.50(74.00 \\
203.50)\end{array}$ & \\
\hline Range & $8.00-743.00$ & $30.00-360.00$ & $11.00-415.00$ & $18.00-370.00$ & $23.00-525.00$ & $8.00-743.00$ & \\
\hline
\end{tabular}

RASS, richmond agitation sedation scale; SD, standard deviation. 
preoperatively compared to those who did not, with a 0.57 relative risk [24]. With this understanding and based on the results of this study, the optimal timing of gabapentin administration for nausea, vomiting, and urinary retention is more than $4 \mathrm{~h}$ prior to the start of surgery.

\section{Research implications}

This research study indicates that the risk of postoperative somnolence and respiratory depression may be low in gynecologic surgery patients and that the timing of administration of gabapentin has a minimal effect on postoperative outcomes with the exception of nausea, vomiting, and urinary retention. This study supports the osteopathic theory that the body is a unit and that all systems are interrelated. Optimizing a patient's preoperative milieu will promote postoperative recovery through support of self-healing and self-regulation. Further research can be conducted to study the optimal timing of gabapentin administration relative to the surgical indication, the procedure performed, and whether the individual patients have a chronic pain history.

\section{Strengths and limitations}

This is the first study to analyze the effects of the timing of preoperative gabapentin administration on postoperative clinical outcomes in gynecologic surgeries. This study analyzed all major gynecologic procedures, indications, and types of procedure, enabling generalizability in gynecologic surgery as a whole.

This study has limitations that should be considered, including the inherent bias of a retrospective study design. This study relied upon perioperative staff to monitor and record patient outcomes, meaning that this is prone to misclassification error and general variability in how different staff members assess patient outcomes. However, because this limitation effects all patients equally in the study, the main outcomes can still be compared across the two groups. This bias is further decreased because the study was conducted at a single institution with standard training and education of perioperative staff. Further, in the $4 \mathrm{~h}$ or more group, the patients reported the reported time of gabapentin administration because they took gabapentin prior to arrival for surgery. This could be limited by the recall bias of the patients. Additionally, the results are undoubtedly impacted by confounding variables that would be better accounted for with future prospective, randomized controlled trials.

\section{Conclusions}

Administration of gabapentin less than $4 \mathrm{~h}$ or greater than $4 \mathrm{~h}$ preoperatively does not significantly affect postoperative somnolence or respiratory depression. Those who took gabapentin four or more hours prior to surgery had significantly less adverse outcomes of nausea/vomiting and urinary retention as compared to those who took it within $4 \mathrm{~h}$ of surgery.

Research funding: None reported.

Author contributions: All authors provided substantial contributions to conception and design, acquisition of data, or analysis and interpretation of data; all authors drafted the article or revised it critically for important intellectual content; all authors gave final approval of the version of the article to be published; and all authors agree to be accountable for all aspects of the work in ensuring that questions related to the accuracy or integrity of any part of the work are appropriately investigated and resolved.

Competing interests: None reported.

Ethical approval: The Mayo Clinic Institutional Review Board (IRB number: 20-003782) deemed our study exempt.

\section{References}

1. Ljungqvist 0 , Scott M, Fearon KC. Enhanced recovery after surgery: a review. JAMA Surg 2017;152:292-8.

2. Bordoni B, Escher AR Jr. Osteopathic principles: the inspiration of every science is its change. Cureus 2021;13:e12478.

3. Nelson G, Kalogera E, Dowdy SC. Enhanced recovery pathways in gynecologic oncology. Gynecol Oncol 2014;135:586-94.

4. Simpson JC, Bao X, Agarwala A. Pain management in enhanced recovery after surgery (ERAS) protocols. Clin Colon Rectal Surg 2019;32:121-8.

5. Enhanced recovery after surgery. Aana.com. https://www.aana. com/practice/clinical-practice-resources/enhanced-recoveryafter-surgery [Accessed 13 Dec 2021].

6. Nelson G, Bakkum-Gamez J, Kalogera E, Glaser G, Altman A, Meyer LA, et al. Guidelines for perioperative care in gynecologic/ oncology: Enhanced Recovery After Surgery (ERAS) Society recommendations-2019 update. Int J Gynecol Cancer 2019;29: 651-68.

7. Alayed N, Alghanaim N, Tan X, Tulandi T. Preemptive use of gabapentin in abdominal hysterectomy: a systematic review and meta-analysis. Obstet Gynecol 2014;123:1221-9.

8. Frouzanfard F, Fazel MR, Abolhasani A, Fakharian E, Mousavi G, Moravveji A. Effects of gabapentin on pain and opioid consumption after abdominal hysterectomy. Pain Res Manag 2013;18:94-6.

9. Ho KY, Gan TJ, Habib AS. Gabapentin and postoperative pain-a systematic review of randomized controlled trials. Pain 2006; 126:91-101. 
10. Steinberg AC, Schimpf MO, White AB, Mathews C, Ellington DR, Jeppson $\mathrm{P}$, et al. Preemptive analgesia for postoperative hysterectomy pain control: systematic review and clinical practice guidelines. Am J Obstet Gynecol 2017;217:303-13.e6.

11. Geng Z, Bi H, Zhang D, Xiao C, Song H, Feng Y, et al. The impact of multimodal analgesia based enhanced recovery protocol on quality of recovery after laparoscopic gynecological surgery: a randomized controlled trial. BMC Anesthesiol 2021;21:179.

12. Lehman A, Kemp EV, Brown J, Crane EK, Tait DL, Taylor VD, et al. Pre-emptive non-narcotic pain medication before minimally invasive surgery in gynecologic oncology. J Minim Invasive Gynecol 2021;28:811-6.

13. Mattson J, Thayer M, Mott SL, Lyons YA, Hardy-Fairbanks A, Hill EK. Multimodal perioperative pain protocol for gynecologic laparotomy is associated with reduced hospital length of stay. J Obstet Gynaecol Res 2021;47:1082-9.

14. Tharp AM, Hobron K, Wright T. Gabapentin-related deaths: patterns of abuse and postmortem levels. J Forensic Sci 2019;64:1105-11.

15. Lunn TH, Husted H, Laursen MB, Hansen LT, Kehlet H. Analgesic and sedative effects of perioperative gabapentin in total knee arthroplasty: a randomized, double-blind, placebo-controlled dose-finding study. Pain 2015;156:2438-48.

16. Cavalcante AN, Sprung J, Schroeder DR, Weingarten TN. Multimodal analgesic therapy with gabapentin and its association with postoperative respiratory depression. Anesth Analg 2017;125:141-6.
17. Stone R, Carey E, Fader AN, Fitzgerald J, Hammons L, Nensi A, et al. Enhanced recovery and surgical optimization protocol for minimally invasive gynecologic surgery: an AAGL white paper. J Minim Invasive Gynecol 2021;28:179-203.

18. Tjandrawinata RR, Setiawati E, Putri RS, Yunaidi DA, Amalia F, Susanto LW. Single dose pharmacokinetic equivalence study of two gabapentin preparations in healthy subjects. Drug Des Dev Ther 2014;8:1249-55.

19. Principles of analgesic use in the treatment of acute pain and chronic cancer pain, 2nd edition. American Pain Society. Clin Pharmacol 1990;9:601-12.

20. Guttuso T Jr. Gabapentin's anti-nausea and anti-emetic effects: a review. Exp Brain Res 2014;232:2535-9.

21. Chiu C, Aleshi P, Esserman LJ, Inglis-Arkell C, Yap E, Whitlock EL, et al. Improved analgesia and reduced post-operative nausea and vomiting after implementation of an enhanced recovery after surgery (ERAS) pathway for total mastectomy. BMC Anesthesiol 2018;18:41.

22. Grant MC, Lee H, Page AJ, Hobson D, Wick E, Wu CL. The effect of preoperative gabapentin on postoperative nausea and vomiting: a meta-analysis. Anesth Analg 2016;122:985.

23. Cangemi DJ, Kuo B. Practical perspectives in the treatment of nausea and vomiting. J Clin Gastroenterol 2019;53:170-8.

24. Han C, Kuang MJ, Ma JX, Ma XL. The efficacy of preoperative gabapentin in spinal surgery: a meta-analysis of randomized controlled trials. Pain Physician 2017;20:649-61. 